\title{
BMJ Use of simulation to assess electronic OPen health record safety in the intensive care unit: a pilot study
}

\author{
Christopher A March, David Steiger, ${ }^{1}$ Gretchen Scholl, ${ }^{2}$ Vishnu Mohan, ${ }^{3}$ \\ William R Hersh, ${ }^{3}$ Jeffrey A Gold ${ }^{2}$
}

To cite: March CA, Steiger D, Scholl G, et al. Use of simulation to assess electronic health record safety in the intensive care unit: a pilot study. BMJ Open 2013;3:e002549.

doi:10.1136/bmjopen-2013002549

- Prepublication history for this paper are available online. To view these files please visit the journal online (http://dx.doi.org/10.1136/ bmjopen-2013-002549).

Received 3 January 2013 Revised 13 March 2013 Accepted 18 March 2013

This final article is available for use under the terms of the Creative Commons Attribution Non-Commercial 2.0 Licence; see http://bmjopen.bmj.com

${ }^{1}$ Department of Hospital Medicine, Oregon Health and Science University, Portland, Oregon, USA

${ }^{2}$ Department of Pulmonary and Critical Care Medicine, Oregon Health and Science University, Portland, Oregon, USA

${ }^{3}$ Department of Medical Informatics \& Clinical Epidemiology, Oregon Health and Science University, Portland, Oregon, USA

Correspondence to Dr Jeffrey A Gold; goldje@ohsu.edu

\section{ABSTRACT}

Objective: To establish the role of high-fidelity simulation training to test the efficacy and safety of the electronic health record (EHR)-user interface within the intensive care unit (ICU) environment.

Design: Prospective pilot study.

Setting: Medical ICU in an academic medical centre.

Participants: Postgraduate medical trainees.

Interventions: A 5-day-simulated ICU patient was developed in the EHR including labs, hourly vitals, medication administration, ventilator settings, nursing and notes. Fourteen medical issues requiring recognition and subsequent changes in management were included. Issues were chosen based on their frequency of occurrence within the ICU and their ability to test different aspects of the EHR-user interface. ICU residents, blinded to the presence of medical errors within the case, were provided a sign-out and given 10 min to review the case in the EHR. They then presented the case with their management suggestions to an attending physician. Participants were graded on the number of issues identified. All participants were provided with immediate feedback upon completion of the simulation.

Primary and secondary outcomes: To determine the frequency of error recognition in an EHR simulation. To determine factors associated with improved performance in the simulation.

Results: 38 participants including 9 interns, 10 residents and 19 fellows were tested. The average error recognition rate was $41 \%$ (range $6-73 \%$ ), which increased slightly with the level of training $(35 \%, 41 \%$ and $50 \%$ for interns, residents, and fellows, respectively). Over-sedation was the least-recognised error (16\%); poor glycemic control was most often recognised $(68 \%)$. Only $32 \%$ of the participants recognised inappropriate antibiotic dosing. Performance correlated with the total number of screens used $(\mathrm{p}=0.03)$.

Conclusions: Despite development of comprehensive EHRs, there remain significant gaps in identifying dangerous medical management issues. This gap remains despite high levels of medical training, suggesting that EHR-specific training may be beneficial. Simulation provides a novel tool in order to both identify these gaps as well as foster EHR-specific training.

\section{ARTICLE SUMMARY}

Article focus

- Developing a simulation environment to test ability of providers to recognise medical errors in the EHR.

- To establish the reproducibility of EHR-based simulation testing.

- To understand the types of medical errors/ patient trends which are not recognised by the average user of the EHR.

\section{Key messages}

- Average users of the EHR, irrespective of the level of training, have a poor rate of recognising disturbing trends in patient condition or medical errors.

- Simulation testing will allow for a structured way to both restructure EHR education as well as redesign.

- Issues related to the EHR-user interface are magnified by the data-rich intensive care unit environment.

Strengths and limitations of this study

- The study demonstrates the feasibility of using EHR simulation to identify patient safety and quality issues related to the EHR/user interface.

- The study provides a framework to test how new educational techniques or EHR interface design can improve patient safety and error recognition.

- This pilot study does not address whether participation in the simulation itself improves the provider use of the EHR.

\section{INTRODUCTION}

Use of the electronic health record (EHR) is growing in the USA, spurred by financial incentives from the American Recovery and Reinvestment Act (ARRA). ${ }^{1}{ }^{2}$ A growing body of research demonstrates that EHRs provide a myriad of benefits, including increased adherence to guideline-based care, decreased prescribing errors and improved disease monitoring. ${ }^{3-5}$ There has been a significant rise in EHR use across the country, with a near tripling in the number of 
hospitals using any form of EHR during the first decade of the 21st century. ${ }^{6}$ By the end of 2011, EHR adoption had increased to over $50 \%$ of all US physicians, stimulated by $\$ 2.5$ billion in incentives paid out under the Health Information Technology for Clinical and Economic Health (HITECH) Act of ARRA. ${ }^{8}$ As even more healthcare systems transition to EHRs, there will be an increasing need for the development of new methods to effectively train healthcare providers, particularly with respect to maximising the functionality of the EHR as a clinical tool.

While EHRs can offer significant benefits, they can also foster errors in ways that paper documentation did not, a phenomenon that has been termed 'e-iatrogenesis'. 9 At the most fundamental level, EHR software itself can be poorly designed and may promote errors such as radiation overdosing or miscalculating patient medication doses. ${ }^{10}$ Medication ordering and monitoring appear to be particularly vulnerable to errors in the EHR. Duplicate medication orders, as well as drug dosing and monitoring errors have been shown to increase in the post-EHR era. ${ }^{11}{ }^{12}$ More complex types of errors arise from the way clinicians interface with the EHR; many of these errors were unforeseen prior to implementation of these systems. ${ }^{13}$ The complexity of EHR implementations has often led to unintended consequences and errors and recent studies have evaluated the concept of fragmentation of the 'big picture' of a patient's trajectory by the vast amount of information displayed in a patient's electronic record and the resultant data overload inflicted on the clinician's cognitive process. $^{14} 15$

In November 2011, the Institute of Medicine (IOM) released a report on the safety of health information technology (HIT) ${ }^{16}$ that detailed challenges associated with the safe implementation of HIT. The report documented both the predictable and the unintended consequences of EHRs. This report also developed a taxonomy for classification of errors with categories that included data fragmentation, over-completeness (including excessive redundancy and copy-and-paste), errors in data recognition and perhaps most importantly, cognitive errors. ${ }^{1617}$ The latter arise when users are unable to effectively process data to make appropriate decisions due to the method by which data are presented within the EHR. $^{18}$

These safety issues are perhaps most relevant in the intensive care unit (ICU), where a $24 \mathrm{~h}$ cycle typically generates over 1300 new data points in the health record for an average patient. ${ }^{19}$ Many of the reports of increased errors, patient morbidity and the failure to successfully implement EHRs have come from the ICU environment. $^{2021}$ In an attempt to address this problem, Ahmed et al described a new EHR interface for their ICU designed to present data in a context-specific and streamlined manner. It was successful at reducing both the total amount of errors per provider and the 'taskload' index, an indirect measure of data overload. ${ }^{22}$
Unfortunately, most institutions do not have the expertise or resources to design their own EHR interface, instead relying on commercial systems.

Adequately training providers is a key component which may improve EHR safety. Studies document that physician training in EHR use is currently suboptimal. Underwood et al demonstrated that while at least 35 days of training was required for physicians to report the highest levels of satisfaction, nearly half the physicians studied $(49.3 \%)$ revealed that they had received three or fewer days of training. Interestingly, respondent ratings on the ease of use for meaningful use measures continued to improve with more than 2 weeks of training. ${ }^{23}$ The IOM and the American Medical Informatics Association (AMIA) have identified EHR development, implementation and training as key areas for new research to improve healthcare quality and safety. ${ }^{16} 24$

In spite of the growth of medical simulation and the increasing emphasis on high-fidelity simulation, little has been performed with EHR-specific simulation training. Simulation training is particularly attractive as it conveys no risk to patients, maintains patient privacy and allows a highly specific and reproducible training environment that can be tailored to the needs of learners and healthcare organisations. ${ }^{25}$ In order for full task training via simulation to be effective, however, there must be specific attention given to creating psychological and functional fidelity, that is, recreating the true 'feel' of the goal environment. ${ }^{26}$ The few studies on EHR simulation have not been in the ICU nor have they truly tested physician ability to recognise and process information (as opposed to order entry). ${ }^{27} 28$ Barnato et al were successful in creating a realistic simulated ICU environment to test decision-making variability in patient triage. ${ }^{29}$ However, in their study, the EHR was utilised as a tool within the simulation as opposed to the focus of the simulation exercise itself.

The goal of our study was to create a highly realistic and complex-simulated ICU patient encounter in the EHR. We developed this simulation as a pilot as part of a longer term goal to teach effective use of the EHR in the ICU to identify common EHR error types such as medication monitoring errors and failure to identify concerning trends in laboratory or vital statistics data, and to help physicians cope with data fragmentation/ overload.

\section{METHODS}

\section{Ethics statement}

The study was approved by the Oregon Health and Science University Institutional Review Board (IRB). The study was deemed minimal risk and informed consent was not required. All participants were provided an IRB-approved information sheet about the protocol. All data were de-identified and stored in a secure file. The authors are willing to share any and all data 
obtained from this research. They will be available via email to the corresponding author.

For the study, a new training environment was created within our enterprise-wide EHR (EPIC Care; Epic Systems, Madison, Wisconsin, USA) that allowed the generation of patient cases with multiple consecutive days of patient data. This was in contrast to the previous training environment that supported only single-day encounters as all data were deleted at the end of each day. The new training environment was an exact replica of the physician's current practice environment; any user-specific settings and customisations generated in actual patient care were retained in the simulation environment (eg, individual preference lists, screen view settings, etc).

Within this new environment, we created a multidaysimulated Medical ICU (MICU) patient case, which detailed the clinical course of a 74-year-old patient with diabetics admitted in septic shock with resulting acute renal failure and acute respiratory distress syndrome (ARDS) requiring mechanical ventilation. The patient improved clinically over the initial $48 \mathrm{~h}$, including resolution of renal failure, shock and fever. Recurrent sepsis developed on the fifth hospital day, presumably due to an inadequate antibiotic dose in the setting of normalisation of renal function. The case was made as robust as possible and included hourly vital signs, a full medication administration report (MAR) including as-needed (PRN) medications, a detailed hourly intake/output report, and nursing, resident, attending and respiratory therapy notes.

The case was designed with the central theme of determining whether a diagnosis of recurrent sepsis would be made. We chose sepsis as the focus because of its high prevalence (it is the leading cause of death in the ICU), the fact that a significant percentage of physicians believe that this diagnosis is missed in patients and epidemiological studies that suggest many patients experience a delay in diagnosis which is associated with worse outcomes. ${ }^{30} 31$ Aside from the physiological and laboratory data associated with the diagnosis, we built in additional errors which we identified after integrating discussion of EHR use into our weekly MICU Morbidity and Mortality conference as occurring at a high frequency. The total number of errors/patient trends within the case was typical for patients with significant missed clinical deterioration, particularly in those cases where clinical decision-making did not meet best practices. In total, 14 individual action items were built in the case that could be grouped into the following three categories: (1) dangerous trends in lab results or vital signs (eg, 25\% reduction in blood pressure with tachycardia and leukocytosis), (2) clear medication errors (eg, incorrect antibiotic dose for renal function) and (3) failure to adhere to institutional or national best practices across critical care (eg, attention to items that are covered by the 'FAST HUG' (Feeding, Analgesia, Sedation, Thromboembolic prevention, Ulcer prophylaxis, Head of Bed elevation and Glycemic Control)). ${ }^{32}$ Table 1 presents a complete list with definitions of the errors included in the case as well as the type of error occurring at the EHR-user interface representing each specific item and specifically in relation to our institution's specific EHR.

The simulated case was then deployed on an EHR workstation in the MICU. Participants included interns, residents (predominantly internal medicine trainees), as

Table 1 Fourteen errors developed throughout the 5-day ICU course

\begin{tabular}{|c|c|}
\hline Error safety issue & EHR category \\
\hline \multicolumn{2}{|l|}{ Changes in patient condition } \\
\hline $25 \%$ Drop in mean arterial pressure, $25 \%$ increase in heart rate & Structure and time, cognition and customisation \\
\hline Recurrent sepsis & Cognition \\
\hline Increasing plateau pressure to $>30$ & Overcompleteness, data finding \\
\hline Increase in $\mathrm{WBC}^{*}$ & Structure and time, cognition and customisation \\
\hline New fever & Structure and time, cognition and customisation \\
\hline \multicolumn{2}{|l|}{ Medication errors } \\
\hline Inappropriate antibiotic dose (2) & Data finding, cognition \\
\hline Low antibiotic trough & Data finding, cognition \\
\hline Use of D5W in hyperglycemic patient & Data finding and overcompleteness \\
\hline \multicolumn{2}{|l|}{ Failure to adhere to best practice } \\
\hline Glucose $>200$ mg/dl & Overcompleteness and data finding \\
\hline Tidal volume of $8 \mathrm{cc} / \mathrm{kg}$ IBW in acute respiratory distress syndrome & Data finding and cognition \\
\hline Over-sedation & Data finding \\
\hline Lack of daily awakenings & Data finding \\
\hline Recognition of fluid balance & Data finding \\
\hline
\end{tabular}


well as pulmonary, medical and anesthesia critical care fellows (of all years of training). All participants had received institution-specific training with our EHR and had already been users of the system prior to testing. This training was standard for all residents and fellows at the beginning of their training and comprised of 1.5 days of small group instruction with one of the institution-dedicated EHR trainers. Training involved hands on use with the system and included tasks such as data retrieval, data entry and instructions on customisation. Users were expected to complete a set number of tasks in each of these areas prior to completion. Each participant was provided a one-page description of the patient, including a brief synopsis of the history and a current physical examination for the context. Participants were told to analyse patient data in order to prepare to 'sign-out' the patient to a colleague, including any management changes they would recommend making to the patient's care. Participants were blinded to the presence of known errors built in the case. Each participant used their own login credentials, which allowed their own personal EHR customisations to be activated within the EHR, and was allotted $10 \mathrm{~min}$ of chart review time which represents the approximate amount of time the average resident spends reviewing the chart while prerounding on an individual patient at our institution. Of note, we initially tested the case with two senior critical care fellows to ensure both its realism (in terms of data presentation) and feasibility of completion in the allotted time.

During the exercise, participants were directly observed by a member of the study team and all data recorded on a standardised data collection sheet. The observer noted both the absolute number of screens used in reviewing the patient record as well as the use of either of two 'high-yield' screens. One of these screens (the 'MD Index' screen) was a gateway into multiple different modes of data presentation, while the other (the 'Synopsis' screen) presented a graphical view of vital sign trends alongside timed MAR and lab data. Of note, while all of our primary data and portal screens were designed to be used within the ICU environment, none are specific to the ICU and they are utilised throughout the inpatient environment.

Each participant made a brief presentation to a member of the study team with specific focus on action items that should be addressed. The presentation was structured to mimic the workflow on daily rounds. Participants were scored based on whether they identified the action items/ clinical trends within the case. Upon the conclusion of the encounter, all participants were given immediate feedback on which issues were correct, which were missed, and where to find the missing data in the EHR.

Differences between groups were analysed using a twotailed student $\mathrm{t}$ test. Correlations were analysed via Spearman's test. (For both, a $p$ value $<0.05$ was considered significant.) All data were analysed with GraphPad Prism (San Diego, California, USA).
$\%$ Correct By Year

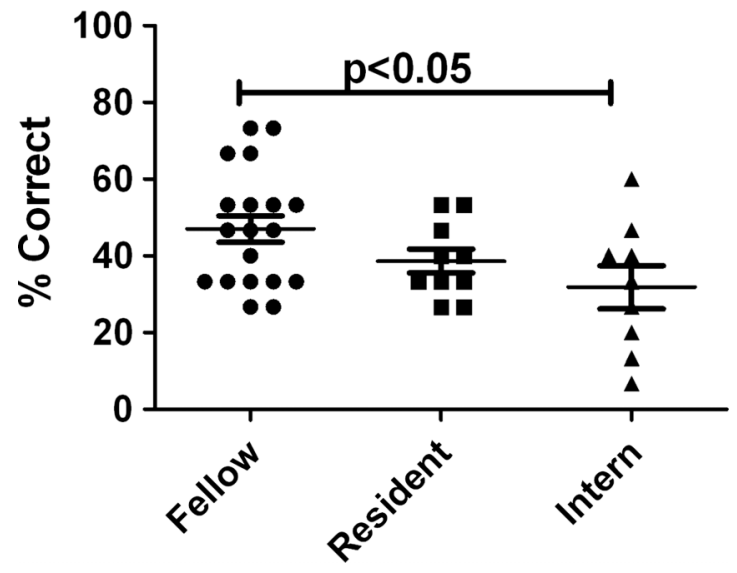

Figure 1 Simulation performance is loosely correlated with the level of training. Thirty-nine participants underwent EHR simulation and graded according to the number of correctly identified errors. Data analysed by analysis of variance.

\section{RESULTS}

A total of 38 participants were tested: 19 fellows, 10 residents and 9 interns. Of the 14 possible medical issues requiring recognition and alteration in management, an average of $41 \%$ (range $6-73 \%$ ) were identified (figure 1). The recognition rate increased significantly with the level of clinical training: interns, residents and fellows recognised $35 \%, 41 \%$ and $50 \%$, respectively ( $\mathrm{p}=0.03$; figure 1 ).

Overall, there was little consistency in the type of errors missed across the cohort as a whole. The least recognised issues were the over-sedation of the patient, and the lack of daily awakenings $(16 \%)$, the latter of which was indicated by a Motor Activity Assessment Scale (MAAS) score varying between zero (unresponsive to noxious stimuli) and one (responsive only to noxious stimuli) ${ }^{33}$ Poor glycemic control was identified but at a relatively low rate $(68 \%$; figure 2$)$. Of greater concern, only $29 \%$ correctly recognised the change in vital signs consistent with recurrent sepsis.

Of note, during the first round of testing, we inadvertently introduced an additional error into the laboratory screen when we built the simulated case. The patient, instead of having $20 \%$ band forms in their manual differential, had $20 \%$ basophils. Only 1 of the 14 people noted this abnormality, providing additional evidence for the potential for the simulation to assess juxtaposition errors as well the extent to which they exist. Finally, except for recognition of an excessive tidal volume $(>6 \mathrm{cc} / \mathrm{kg})(58 \%$ vs $21 \% ; \mathrm{p}=0.045)$ and lack of daily awakenings ( $53 \%$ vs $16 \%$; $\mathrm{p}=0.038$ ), two best practices for intubated patients with ARDS, ${ }^{34} 35$ there were no statistical differences between fellows and residents in recognition of other errors or safety issues (figure 3). Overall, the average participant visited 16.4 different screens (an average of $35.6 \mathrm{~s}$ per screen). The number of individual screens visited correlated with the number of errors recognised (figure 4). 


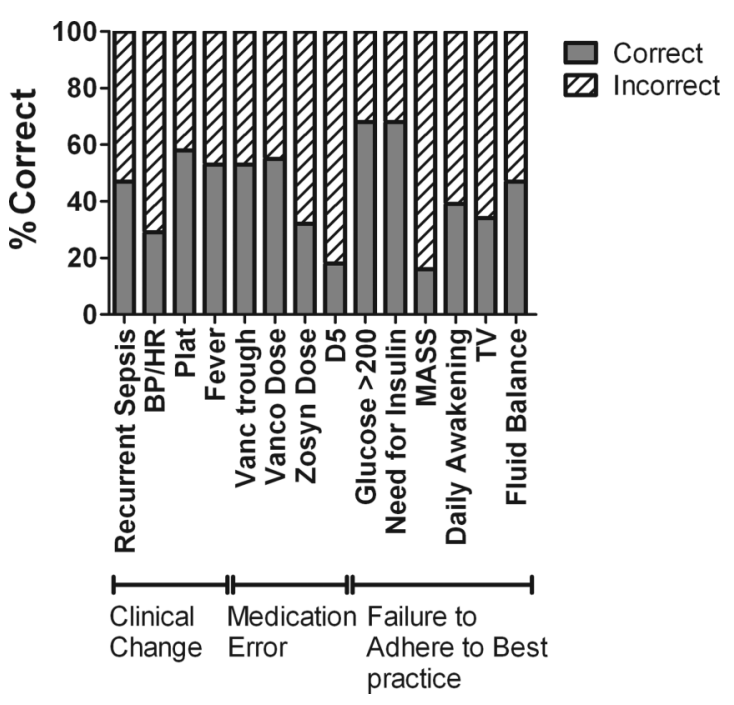

Figure 2 Frequency of error recognition. The number of participants correctly identifying each of the 14 main errors built into the simulation.

We also looked at whether viewing 'high impact' data screens impacted the ability of participants to find errors. We looked specifically at the two main portal pages within our EHR. One was the 'Synopsis' page that presents haemodynamics in a graphical format as well as all medications and lab values. The other was the 'MD Index,' a portal, created by our institution as part of its customisation of the EHR, which allows easy access to a number of different data screens, including vitals, MAR, hemodynamics. We found that use of the Synopsis screen was associated with lower performance on the simulation. Conversely, use of the MD Index was associated with a significantly better use of the system (figure 5).

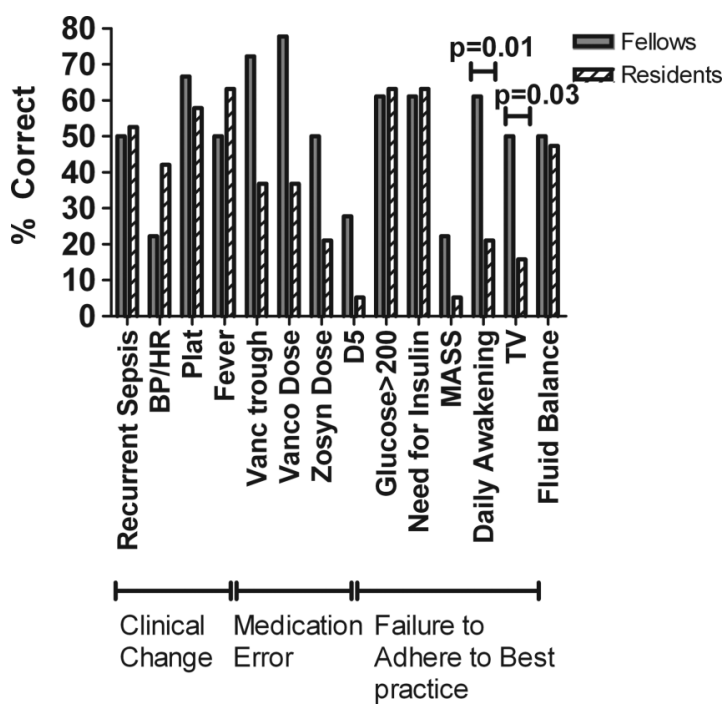

Figure 3 Successful error recognition is mostly independent of the training level. Overall recognition rate by fellows (blue) and residents (red) for each of the 14 major errors. Data analysed by t test.

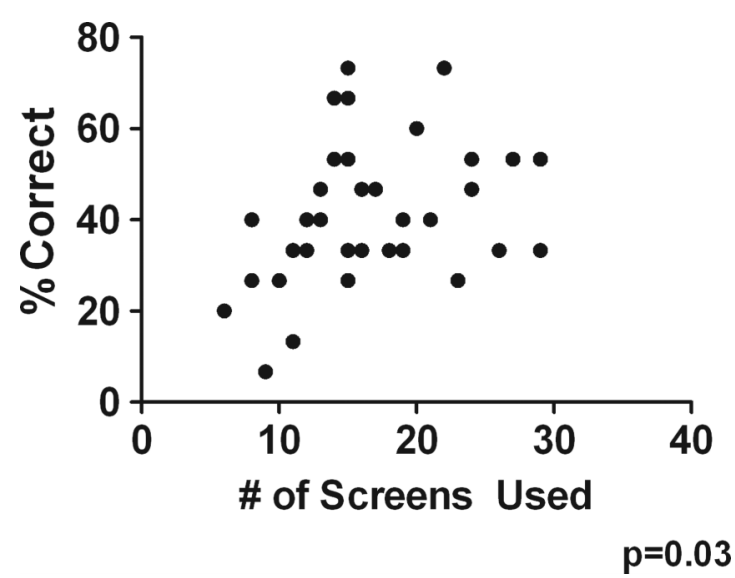

Figure 4 Increased screen utilisation is associated with improved performance. The number of independent screens visited was correlated with the overall performance on simulation.

\section{DISCUSSION}

In this pilot study, we developed and used a novel ICU-specific EHR simulation based on a commonly used commercial system. There is an increasing trend to use simulation as a tool for assessing end-user competency and improving patient safety. A high-fidelity simulation allows our study to be conducted in an authentic and realistic clinical environment, with the opportunity to provide the participant with immediate feedback at the conclusion of the simulation. Since end-users often customise their 'user interface' quite significantly, we felt that it was important to create a simulation environment for our participants that was identical to the actual production EHR environment, including the log-in and key clinical screens, and maintain any customisation that end-users had already developed. Second, the simulation is performed in the ICU on existing clinical workstations further enhancing environmental fidelity. Third, the case is based on an actual ICU patient and data were representative of a typical high-complexity ICU patient in terms of the quality, the amount of data within the patient chart (including the fact that this was a 5-day ICU stay) and the types of errors and safety issues
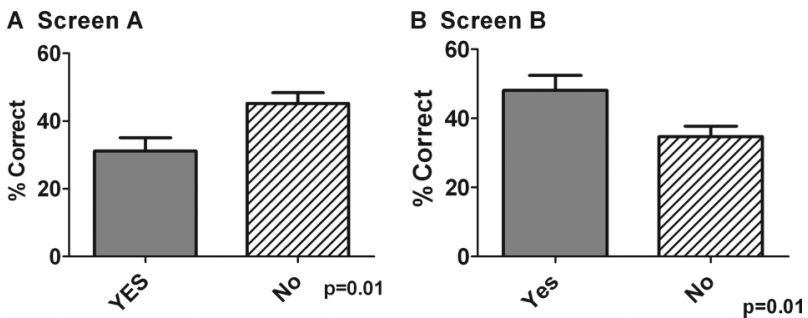

Figure 5 Individual screen use correlates with performance. The overall success rate was tabulated for user of two of the major portals; screens A and B. Overall, use of screen A was associated with increased error recognition while screen $B$ use was associated with poor performance. Data analysed via t test. 
typically encountered in our ICU. Fourth is our method of assessment. By having participants present the patient to an ICU physician (either attending or senior fellow), we created an environment consistent with our existing workflow (as opposed to answering specific questions on a written examination, using surveys to elicit information, or recounting the simulation after the passage of much time). Finally, the timed nature of the exercise was much more consistent with the real workflow in an ICU where physicians only have a limited time to search for data and was consistent with the existing workflow within our ICU.

Our findings were both surprising and concerning. First, only $41.5 \%$ of errors were recognised, and while fellows performed statistically significantly better than interns or residents, their overall performance was still below what most would consider acceptable (47\%). Further, the most severe errors, such as development of impending shock, were recognised at even a lower frequency $(40 \%)$. We observed the overall poor performance among the members of all levels of training, despite all of the participants having received general training with our EHR and over a years' use with the system. Given this finding, it appears that a major stumbling block is the physician interface with the EHR as opposed to a pure knowledge deficit. However, these observations appear to be in-line with the reported literature. Nearly $89 \%$ of physicians believe the diagnosis of sepsis is missed in the inpatient setting. ${ }^{31}$ In patients with ARDS, as in this case, nearly $70 \%$ of patients are still not managed with appropriate ventilator strategies. ${ }^{34}$ Medication errors, including inappropriate dosing due to changing renal function, account for nearly $78 \%$ of the total reported errors in the ICU. Finally, nearly $40 \%$ of ICU patients are oversedated without acknowledgement of their sedation score. ${ }^{35} 36$ Finally, amongst patients who have in-hospital cardiac arrest or need for ICU admission, nearly $60 \%$ have evidence of clinical decompensation prior to transfer and in one study, medical staff were only aware of all of the physiologic abnormalities in $34 \%$ of patients. ${ }^{37} 38$

Our findings are consistent with the description of others detailing the ICU as a vulnerable environment for the EHR. For example, Han et al documented an increased mortality with the introduction of computerised provider order entry (CPOE) into their Neonatal ICU. ${ }^{21}$ This was believed not to be due to the system itself, but rather due to poor implementation of the system, lack of customisation, poor workflow and overall poor education and training on how to manage the physician-EHR interface. This assessment was supported by a subsequent study documenting improved outcomes with implementation of an identical system in a similar style ICU. ${ }^{39}{ }^{40}$ A similar experience was observed at another institution, where an enterprise-wide EHR implementation of their EHR proved to be successful, with the exception of the MICU. ${ }^{20}$ MICU-specific problems were attributed to poor training, inadequacies in the EHR-physician interface and lack of customisation creating unmanageable workflow issues, and the system was taken off-line within 6 months. Only after improved customisation, increasing the number of available computers and improved training and education, were they able to safely re-introduce the system into their ICU. ${ }^{20}$

While the concept of patient-based simulation in general is not new, our study is one of the first to use robust, high-fidelity simulation to objectively assess successful use of the EHR and to specifically target identification of changes in the clinical status as the primary endpoint. When EHRs have been utilised in simulation training, it has often been used with non-physicians such as pharmacy or PA students, or rather included in a broader simulation exercise where little emphasis was placed on the interface with the EHR itself. ${ }^{27}{ }^{41}$ Interestingly, a recent set of studies from one group has used a combination of simulated cases and video analysis to assist in EHR design. ${ }^{42}$ However, these studies focused on CPOE (as opposed to the other functions of EHRs including data retrieval) and no data were provided as to the fidelity of the simulation or the clinical context of the actual cases.

Within the ICU, two studies have specifically addressed the use of EHR simulation. In one, physicians were tested about their decision-making with regard to end-of-life care in a virtual patient admitted to the ICU with metastatic cancer and septic shock. In this scenario, the EHR was utilised as a tool for disseminating the casebased information while efficient and appropriate use of the EHR was not assessed. ${ }^{29}$ In the second, researchers hypothesised that the user interface to their existing EHR decreased efficiency with the system and impaired data finding and increased cognitive errors. They had 20 providers review a case in both their original EHR and one with a new front-end to improve data finding, with participants answering eight specific questions specifically related to the management of a bleeding patient. ${ }^{22}$ The new EHR significantly reduced the number of incorrect answers to the questions overall, although for one question focusing on medications, errors increased. This study did have several limitations, including the failure to use a high-fidelity environment (use of a testing room), failure to test efficiency with the system (no apparent time limit), a very directed set of questions to answer to assess data finding (as opposed to the more fluid unknown situation of the average ICU patient) and failure to test longitudinal evaluation of data past $24 \mathrm{~h}$.

The results of our pilot study significantly expand upon these prior studies and will allow us to design a more robust educational and quality improvement initiative around EHR simulation. First, we now have a blueprint for the creation of additional cases, a prerequisite to determine the impact of participation in the simulation. Second, we have established baseline error recognition rates for users at all levels of training and experience, thus allowing us to adequately determine the sample size required for additional studies. For 
example, based on the data from cardiac arrest simulation, we can expect that participation in this exercise results in a nearly $20 \%$ improvement in error recognition on repeat testing, ${ }^{43}$ thus requiring at least 10 participants at each level of training to undergo repeat testing with additional cases to establish this hypothesis. Finally, by establishing baseline usability data and simulation infrastructure, we now have the ability to also test the effect of alterations in the EHR user interface on error recognition and overall performance.

It is important to acknowledge several limitations of our study. First, we only tested data retrieval in this part of the simulation. We recognise that the EHR affects multiple aspects of delivery of care, including communication and order entry. However, the process of data retrieval, process and recognition is the foundation for effective communication and order entry and thus we felt a logical place to begin. We plan to expand this simulation to address these aspects of the EHR in the future. Second the nature and the number of errors built in the case. We have discovered, through the incorporation of the EHR into our weekly Morbidity and Mortality conference, that clinical deterioration in patients is often heralded by numerous clinical clues and is often caused by a number of small errors within an individual case both cognitive and system related. ${ }^{44}$ It is not uncommon for a patient with nosocomial clinical deterioration, as in this case, to have this number of issues that need to be identified. However, we also acknowledge that care of the average ICU patient involves an interprofessional team of pharmacists, nurses and respiratory therapists. As a result, until our simulation is disseminated to all members of the team simultaneously, we cannot be certain that every missed issue by the physician will not be caught by other members of the team and thus result in direct patient harm. Further, it should be stressed that the goal of the simulation is to test the system under high-stress/dangerous situations. We believe this is not only a unique aspect in our study, but is essential to ensure that the system works optimally under all clinical situations. Third, we acknowledge that the case created is unique to the ICU environment. However, we believe with appropriate case creation, the same type of simulation can be used successfully in any clinical care environment. Fourth, while the case itself was realistic in terms of data presentation and the testing was performed in situ, participants were still aware that this was a simulated case. As a result, there could still exist a significant Hawthorne effect resulting in an overestimation of the error recognition rate. Finally, the studies were performed utilising one specific EHR (EPIC Care). While the most commonly used EHR by US physicians, we acknowledge that each EHR and user interface will have its own strengths and weaknesses in terms of data recognition or processing. ${ }^{45}$ However, our methods using robust and realistic cases will allow other researchers to test the functionality of any other EHR.
In conclusion, implementation of EHRs has brought a massive amount of information to the fingertips of ICU practitioners across the country. This study demonstrates that the combination of sheer data and provider knowledge is not sufficient for quality patient care: utilisation of the EHR is a skill that must be learned. There is much room for improvement both in the interface itself and how we teach its use. Through the creation of standardised cases for EHR simulation, we now have the infrastructure to improve user education as well as objectively test the efficacy of both new educational techniques and EHR redesign.

Contributors CAM helped design the protocol and conducted the simulation experiments. DS conducted the simulation experiments and helped with data analysis. JAG designed the study, performed the simulations and is primarily responsible for data analysis and is the guarantor. GS was responsible for technical aspects of the design of the simulation environment. VM and WRM were responsible for both study design and data analysis. All authors have read and approved the final manuscript.

Funding NIH (grant number 1U240C000015) and AHRQ (grant number R18 HS 021637-02).

\section{Competing interests None.}

Ethics approval OHSU Institutional Review Board.

Provenance and peer review Not commissioned; externally peer reviewed.

Data sharing statement No additional data are available.

\section{REFERENCES}

1. Blumenthal D. Launching HITECH. N Engl J Med 2010;362:382-5

2. Blumenthal D. Implementation of the federal health information technology initiative. N Engl J Med 2011;365:2426-31.

3. Chaudhry B, Wang J, Wu S, et al. Systematic review: impact of health information technology on quality, efficiency, and costs of medical care. Ann Intern Med 2006;144:742-52.

4. Goldzweig CL, Towfigh A, Maglione M, et al. Costs and benefits of health information technology: new trends from the literature. Health Aff (Millwood) 2009;28:w282-93.

5. Buntin MB, Burke MF, Hoaglin MC, et al. The benefits of health information technology: a review of the recent literature shows predominantly positive results. Health Aff (Millwood) 2011;30:464-71.

6. DesRoches CM, Worzala C, Joshi MS, et al. Small, nonteaching, and rural hospitals continue to be slow in adopting electronic health record systems. Health Aff (Millwood) 2012;31:1092-9.

7. Jha AK, Ferris TG, Donelan K, et al. How common are electronic health records in the United States? A summary of the evidence. Health Aff (Millwood) 2006;25:w496-507.

8. Hsiao CJ, Hing E, Socey TC, et al. Electronic health record systems and intent to apply for meaningful use incentives among office-based physician practices: United States, 2001-2011. NCHS Data Brief 2011;79:1-8.

9. Weiner JP, Kfuri T, Chan K, et al. "e-latrogenesis": the most critical unintended consequence of CPOE and other HIT. J Am Med Inform Assoc 2007; 14:387-8; discussion 89.

10. Ammenwerth $\mathrm{E}$, Shaw NT. Bad health informatics can kill-is evaluation the answer? Methods Inf Med 2005;44:1-3.

11. Reckmann MH, Westbrook Jl, Koh Y, et al. Does computerized provider order entry reduce prescribing errors for hospital inpatients? A systematic review. J Am Med Inform Assoc 2009;16:613-23.

12. Koppel R, Metlay JP, Cohen A, et al. Role of computerized physician order entry systems in facilitating medication errors. JAMA 2005;293:1197-203.

13. Ash JS, Sittig DF, Dykstra RH, et al. Categorizing the unintended sociotechnical consequences of computerized provider order entry. Int J Med Inform 2007;76(Suppl 1):S21-7.

14. Campbell EM, Sittig DF, Ash JS, et al. Types of unintended consequences related to computerized provider order entry. J Am Med Inform Assoc 2006;13:547-56. 
15. Ash JS, Gorman PN, Lavelle M, et al. Perceptions of physician order entry: results of a cross-site qualitative study. Methods Inf Med 2003;42:313-23.

16. Warden GL, Bagian JP. Health IT and patient safety: builder safer systems for better care. Washington, DC: Institute of Medicine National Academies Press, 2011.

17. Ash JS, Kilo CM, Shapiro M, et al. Roadmap for provision of safer healthcare information systems: preventing e-iatrogensis. Washington, DC: Institute of Medicine, 2011.

18. Berg $\mathrm{M}$, Goorman $\mathrm{E}$. The contextual nature of medical information. Int J Med Inform 1999;56:51-60.

19. Manor-Shulman O, Beyene J, Frndova $\mathrm{H}$, et al. Quantifying the volume of documented clinical information in critical illness. J Crit Care 2008;23:245-50.

20. Ali NA, Mekhjian HS, Kuehn PL, et al. Specificity of computerized physician order entry has a significant effect on the efficiency of workflow for critically ill patients. Crit Care Med 2005;33:110-14

21. Han YY, Carcillo JA, Venkataraman ST, et al. Unexpected increased mortality after implementation of a commercially sold computerized physician order entry system. Pediatrics 2005;116:1506-12.

22. Ahmed A, Chandra S, Herasevich V, et al. The effect of two different electronic health record user interfaces on intensive care provider task load, errors of cognition, and performance. Crit Care Med 2011;39:1626-34.

23. Underwood WS, Brookstone AJ, Barr MS. The correlation of training duration with EHR usability and satisfaction: implications for meaningful use. Am EHR Patners 2011:1-32.

24. Bloomrosen M, Starren J, Lorenzi NM, et al. Anticipating and addressing the unintended consequences of health IT and policy: a report from the AMIA 2009 Health Policy Meeting. J Am Med Inform Assoc 2011;18:82-90.

25. Castanelli DJ. The rise of simulation in technical skills teaching and the implications for training novices in anaesthesia. Anaesth Intensive Care 2009;37:903-10.

26. Maran NJ, Glavin RJ. Low- to high-fidelity simulation-a continuum of medical education? Med Educ 2003;37(Suppl 1):22-8.

27. Muma RD, Niebuhr BR. Simulated patients in an electronic patient record. Acad Med 1997;72:72.

28. Kushniruk AW, Borycki EM, Anderson JG, et al. Preventing technology-induced errors in healthcare: the role of simulation. Stud Health Technol Inform 2009;143:273-6.

29. Barnato $\mathrm{AE}, \mathrm{Hsu} \mathrm{HE}$, Bryce $\mathrm{CL}$, et al. Using simulation to isolate physician variation in intensive care unit admission decision making for critically ill elders with end-stage cancer: a pilot feasibility study. Crit Care Med 2008;36:3156-63.

30. Kumar A, Roberts D, Wood KE, et al. Duration of hypotension before initiation of effective antimicrobial therapy is the critical determinant of survival in human septic shock. Crit Care Med 2006;34:1589-96.
31. Poeze $\mathrm{M}$, Ramsay $\mathrm{G}$, Gerlach $\mathrm{H}$, et al. An international sepsis survey: a study of doctors' knowledge and perception about sepsis. Crit Care 2004;8:R409-13.

32. Vincent JL. Give your patient a fast hug (at least) once a day. Crit Care Med 2005;33:1225-9.

33. Devlin JW, Boleski G, Mlynarek M et al. Motor activity assessment scale: a valid and reliable sedation scale for use with mechanically ventilated patients in an adult surgical intensive care unit. Crit Care Med 1999;27:1271-5.

34. Walkey AJ, Wiener RS. Risk factors for underuse of lung-protective ventilation in acute lung injury. $J$ Crit Care 27:323 e1-9.

35. Devlin JW. The pharmacology of oversedation in mechanically ventilated adults. Curr Opin Crit Care 2008;14:403-7.

36. Camire E, Moyen E, Stelfox HT. Medication errors in critical care: risk factors, prevention and disclosure. CMAJ 2009; 180:936-43

37. Fuhrmann L, Lippert A, Perner A, et al. Incidence, staff awareness and mortality of patients at risk on general wards. Resuscitation 2008;77:325-30.

38. Kause J, Smith G, Prytherch D, et al. A comparison of antecedents to cardiac arrests, deaths and emergency intensive care admissions in Australia and New Zealand, and the United Kingdom-the ACADEMIA study. Resuscitation 2004;62:275-82.

39. Del Beccaro MA, Jeffries HE, Eisenberg MA, et al. Computerized provider order entry implementation: no association with increased mortality rates in an intensive care unit. Pediatrics 2006; 118:290-5.

40. Sittig DF, Ash JS, Zhang J, et al. Lessons from Unexpected increased mortality after implementation of a commercially sold computerized physician order entry system. Pediatrics 2006;118:797-801.

41. Frenzel JE. Using electronic medical records to teach patient-centered care. Am J Pharm Educ 2010;74:71.

42. Borycki EM, Kushniruk AW, Kuwata S, et al. Engineering the electronic health record for safety: a multi-level video-based approach to diagnosing and preventing technology-induced error arising from usability problems. Stud Health Technol Inform 2011;166:197-205.

43. Donoghue AJ, Durbin DR, Nadel FM, et al. Effect of high-fidelity simulation on pediatric advanced life support training in pediatric house staff: a randomized trial. Pediatr Emerg Care 2009;25:139-44.

44. Graber ML, Franklin N, Gordon R. Diagnostic error in interna medicine. Arch Intern Med 2005;165:1493-9.

45. Kane L. EHR Report 2012: Physicians Rank Top EHRs. Medscape: Medscape from Web MD, 2012.

46. Ash JS, Berg M, Coiera E. Some unintended consequences of information technology in health care: the nature of patient care information system-related errors. J Am Med Inform Assoc 2004;11:104-12. 